Provided for non-commercial research and education use. Not for reproduction, distribution or commercial use.

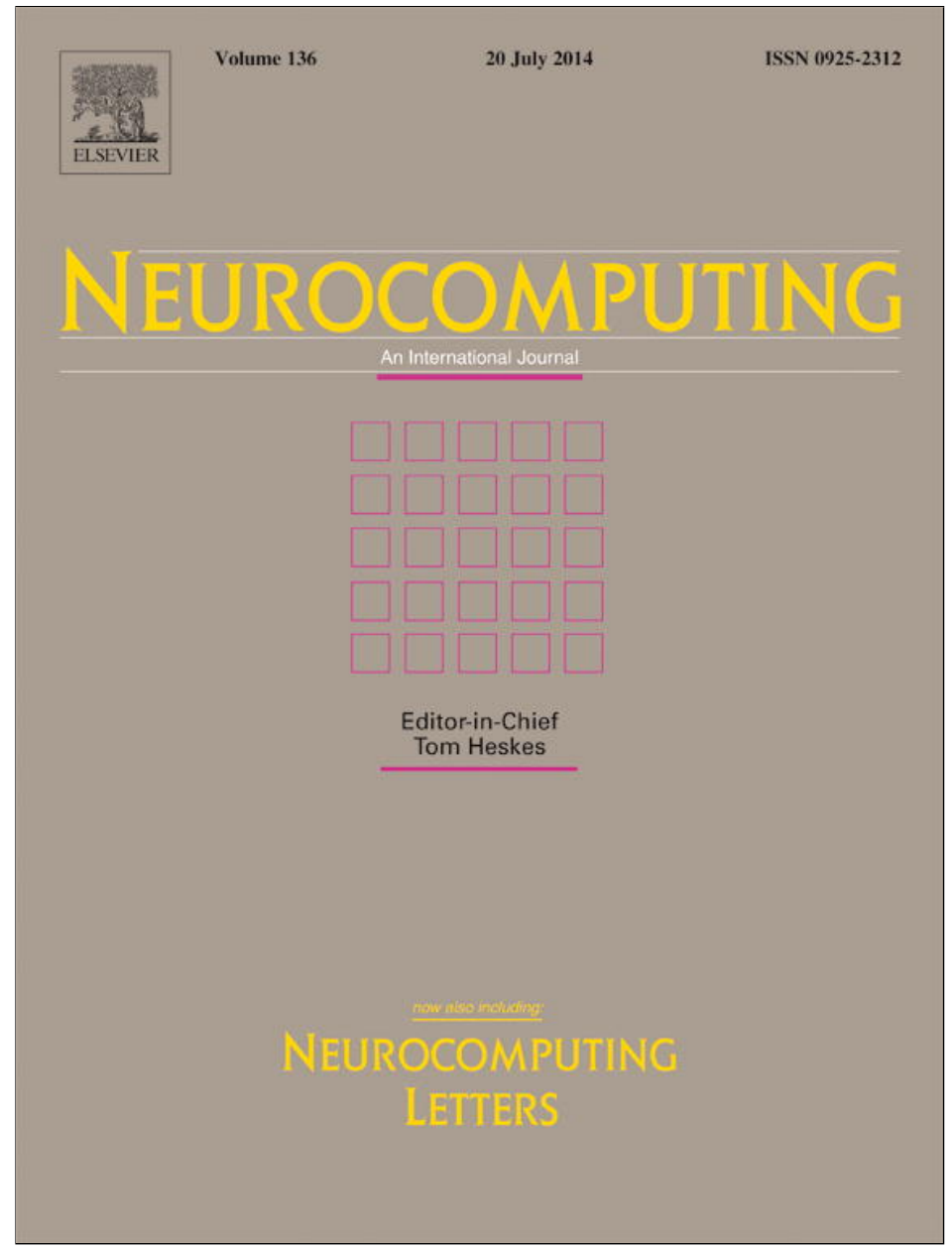

This article appeared in a journal published by Elsevier. The attached copy is furnished to the author for internal non-commercial research and education use, including for instruction at the authors institution and sharing with colleagues.

Other uses, including reproduction and distribution, or selling or licensing copies, or posting to personal, institutional or third party websites are prohibited.

In most cases authors are permitted to post their version of the article (e.g. in Word or Tex form) to their personal website or institutional repository. Authors requiring further information regarding Elsevier's archiving and manuscript policies are encouraged to visit:

http://www.elsevier.com/authorsrights 


\title{
For micro-expression recognition: Database and suggestions
}

\author{
Wen-Jing Yan ${ }^{\mathrm{a}, \mathrm{b}}$, Su-Jing Wang ${ }^{\mathrm{a}}$, Yong-Jin Liu ${ }^{\mathrm{c}}$, Qi Wu ${ }^{\mathrm{d}}$, Xiaolan $\mathrm{Fu}^{\mathrm{a}, *}$ \\ a State Key Laboratory of Brain and Cognitive Science, Institute of Psychology, Chinese Academy of Sciences, Beijing 100101, China \\ ${ }^{\mathrm{b}}$ University of Chinese Academy of Sciences, Beijing 100049, China \\ ${ }^{\mathrm{c}}$ TNList, Department of Computer Science and Technology, Tsinghua University, Beijing 100084, China \\ ${ }^{d}$ Department of Psychology, Hunan Normal University, Changsha 410000, China
}

\section{A R T I C L E I N F O}

\section{Article history:}

Received 8 April 2013

Received in revised form

8 November 2013

Accepted 18 January 2014

Communicated by Qingshan Liu

Available online 15 February 2014

\section{Keywords:}

Micro-expression

Database

Recognition

Suggestion

\begin{abstract}
A B S T R A C T
Micro-expression is gaining more attention in both the scientific field and the mass media. It represents genuine emotions that people try to conceal, thus making it a promising cue for lie detection. Since micro-expressions are considered almost imperceptible to naked eyes, researchers have sought to automatically detect and recognize these fleeting facial expressions to help people make use of such deception cues. However, the lack of well-established micro-expression databases might be the biggest obstacle. Although several databases have been developed, there may exist some problems either in the approach of eliciting micro-expression or the labeling. We built a spontaneous micro-expression database with rigorous frame spotting, AU coding and micro-expression labeling. This paper introduces how the micro-expressions were elicited in a laboratory situation and how the database was built with the guide of psychology. In addition, this paper proposes issues that may help researchers effectively use micro-expression databases and improve micro-expression recognition.
\end{abstract}

(c) 2014 Elsevier B.V. All rights reserved.

\section{Introduction}

Micro-expression is a brief facial movement which reveals an emotion that a person tries to conceal [1,2]. Most notably, TV series Lie to Me brought the idea of micro-expression to the public. The reputation of micro-expression is derived from its potential practical applications in various areas, such as clinical diagnosis, national security and interrogations [3-5] because micro-expression may reveal genuine feelings and help detect lies. Lie detection based on micro-expression is not just a fiction, but stems from scientific researches. Haggard and Isaacs first discovered micro-expression (micro-momentary expression) and considered it as repressed emotions [6,7]. In 1969, Ekman analyzed an interviewing video of a patient stricken with depression who tried to commit suicide and found micro-expressions. From then on, several researches have been conducted in the field of micro-expression but few results were published. Ekman [2] even claimed that micro-expressions might be the most promising approach to detect deception.

Micro-expression is featured by its short duration. Though there is a debate on the duration, the generally accepted upper limit duration is $0.5 \mathrm{~s}[8,9]$. Besides, micro-expression usually occurs with low intensity [8]. Because of the short duration and low intensity, it is usually imperceptible to the naked eyes [1]. To make better use of micro-expression in lie detection, one solution

\footnotetext{
* Corresponding author.

E-mail address: fuxl@psych.ac.cn (X. Fu).
}

is to resort to computers to automatically detect and recognize micro-expressions. An automatic micro-expression recognition system would have far-reaching influence in the fields such as national safety, transportation safety and even clinical diagnosis.

Expression recognition has been intensively studied in the past [10], while little attention was paid to micro-expression recognition until several years ago. Micro-expression recognition raises a great challenge to computer vision because of its short duration and low intensity. The even bigger obstacle is the lack of well-established databases. Recently, several groups have developed micro-expression databases. However, we realize that the existing micro-expression databases have some problems. In the following, we will review the existing micro-expression databases and then introduce CASME database.

This paper is an extended version of our conference paper ${ }^{1}$ [11]. Differently, a further review of the previous databases was given, some new findings of micro-expressions were presented, some challenges were pointed out and some suggestions in automatic micro-expression detection and recognition were provided.

\section{The existing micro-expression databases}

In the following, existing micro-expression databases were reviewed. Table 1 gives a brief description for each database. In USD-HD [12] and Polikovsky's database [13], the drawback is that

\footnotetext{
${ }^{1}$ Part of this work was presented at FG2013.
} 
Table 1

The existing micro-expression databases.

\begin{tabular}{|c|c|c|}
\hline Database & Profile & Problem(s) \\
\hline USF-HD & $\begin{array}{l}\text { It contains } 100 \text { micro-expressions. } \\
\text { Participants were asked to } \\
\text { perform both macro- and micro- } \\
\text { expressions }\end{array}$ & $\begin{array}{l}\text { Posed micro-expressions } \\
\text { rather than spontaneous } \\
\text { ones }\end{array}$ \\
\hline $\begin{array}{r}\text { Polikovsky's } \\
\text { database }\end{array}$ & $\begin{array}{l}\text { It contains } 10 \text { models, who were } \\
\text { instructed to simulate the micro- } \\
\text { expression motion }\end{array}$ & $\begin{array}{l}\text { Posed micro-expressions } \\
\text { rather than spontaneous } \\
\text { ones }\end{array}$ \\
\hline YorkDDT & $\begin{array}{l}\text { It contains } 18 \text { micro-expressions } \\
\text { which were collected from the } \\
\text { recordings in Warren's study [15] }\end{array}$ & $\begin{array}{l}\text { Spontaneous but with other } \\
\text { irrelevant facial movements; } \\
\text { small sample }\end{array}$ \\
\hline $\begin{array}{l}\text { SMIC } \\
\text { database }\end{array}$ & $\begin{array}{l}\text { It contains } 77 \text { spontaneous micro- } \\
\text { expressions which were recorded } \\
\text { by a } 100 \mathrm{fps} \text { camera }\end{array}$ & $\begin{array}{l}\text { Spontaneous micro- } \\
\text { expressions; classified as } \\
\text { positive, negative }\end{array}$ \\
\hline
\end{tabular}

Table 2

Criteria for labeling the emotions and the frequency in the database. ${ }^{a}$

\begin{tabular}{llc}
\hline $\begin{array}{l}\text { Micro- } \\
\text { expression }\end{array}$ & Criteria & $\begin{array}{l}\text { Number of } \\
\text { samples }\end{array}$ \\
\hline Happiness & Either AU6 or AU12 must be present & 9 \\
Sadness & AU1 must be present & 6 \\
Disgust & At least one of AU9, AU10 must be present & 44 \\
Surprise & AU1 +2, AU25 or AU2 must be present & 20 \\
Fear & Either AU1 +2 +4 or AU20 must be present & 2 \\
Repression & AU14, AU15 or AU17 is presented alone or in & 38 \\
Tense & combination & 69 \\
& AU4 or other emotion-related facial & \\
\hline
\end{tabular}

a The emotion labeling is just partly based on the AUs because micro-expressions are usually partial and in low intensity. Therefore, we also take account of participants' self-report and the content of the video episodes.

they consist of posed micro-expressions rather than spontaneous ones. However, micro-expression is considered involuntary and difficult to disguise [1]. In addition, the duration limit set for micro-expressions in USD-HD (2/3 s) is longer than the generally defined one (1/2s). As for YorkDDT [14], the samples are spontaneous micro-expressions with high ecological validity but accompanied with other irrelevant head and face movements when speaking. For the early stages of microexpression recognition, such complicated facial movement is not ideal, as it greatly increases the complexity of the recognition task. Furthermore, very few micro-expressions were acquired in this dataset because micro-expression is difficult to elicit with the "telling lies" approach. SMIC database [14] contains spontaneous micro-expressions elicited in a laboratory. This is a great improvement compared with the posed micro-expression databases. This database, however, did not provide AUs for the samples and the micro-expression labeling was only based on participants' self-report. This might cause a problem since video episodes convey various emotional stimuli and thus an overall report may not be precise (e.g. chewing a worm in a video episode may be disgusting but sometimes also amusing or surprising). Besides, some facial movements may be emotion-irrelevant, such as moving eyebrows due to the changes in eyesight. These irrelevant facial movements should be removed. Based on previous issues and drawbacks, we try to build an improved micro-expression database to facilitate the development of a robust automatic micro-expression recognition system.

\section{CASME database}

\subsection{Database profile}

The Chinese Academy of Sciences Micro-Expression (CASME) database contains 195 spontaneous micro-expressions filmed

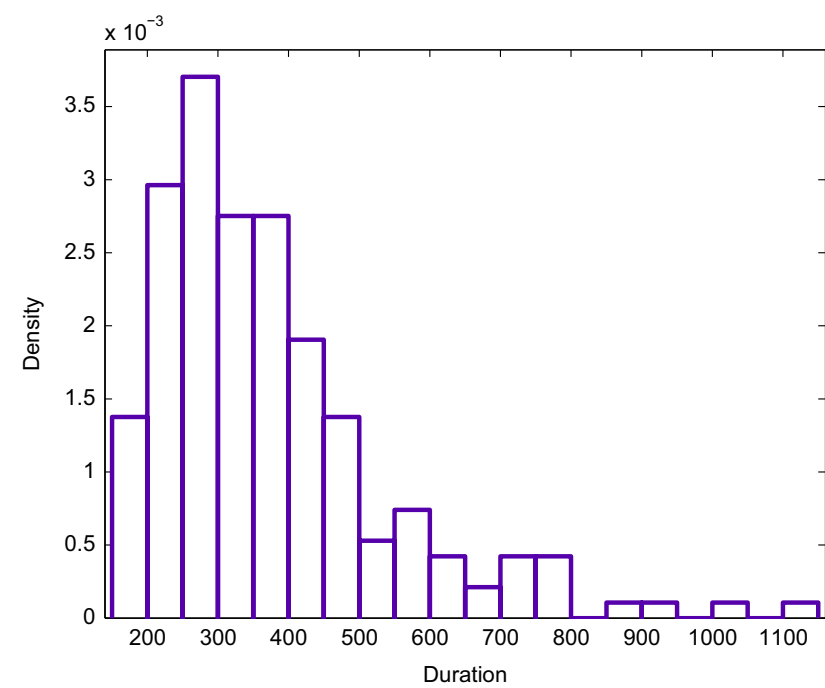

Fig. 1. The distribution of total duration of the micro-expressions.

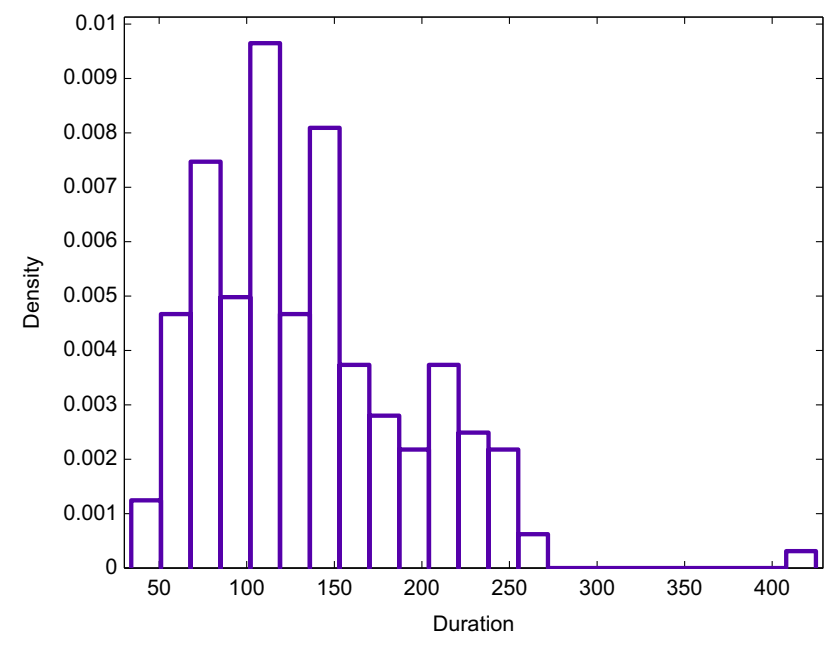

Fig. 2. The distribution of onset duration of the micro-expressions.

under $60 \mathrm{fps}$. These samples were coded so that the onset, peak and offset frames were tagged. The onset frame was the first frame which changes from the baseline (usually neutral facial expressions). The apex- 1 frame is the first frame that reached highest intensity of the facial expression and if it keeps for a certain time, the apex- 2 frame is coded. Facial expressions with the duration no more than $500 \mathrm{~ms}$ were selected for the database. In addition, facial expressions that lasted more than $500 \mathrm{~ms}$ but their onset duration less than $250 \mathrm{~ms}$ were also selected because fast-onset facial expressions are also characterized as micro-expression [8] (that is why the duration of some samples exceed $500 \mathrm{~ms}$ ). The distributions of the samples' duration were provided (see Figs. 1 and 2). Action units (AUs) [16] were marked and emotion labels were given (Fig. 3). To enhance the validity, emotions were labeled based on 3 aspects: AU-combinations, the main emotion of the video episode and participants' report (see Table 3). Compared with other micro-expression databases, the CASME database includes the following advantages:

(1) The samples are spontaneous micro-expressions. The frames before and after each target micro-expression in each video sample show baseline (usually neutral) faces.

(2) Participants were asked to maintain a neutral face (neutralization paradigm) in the study. Therefore, micro-expressions 


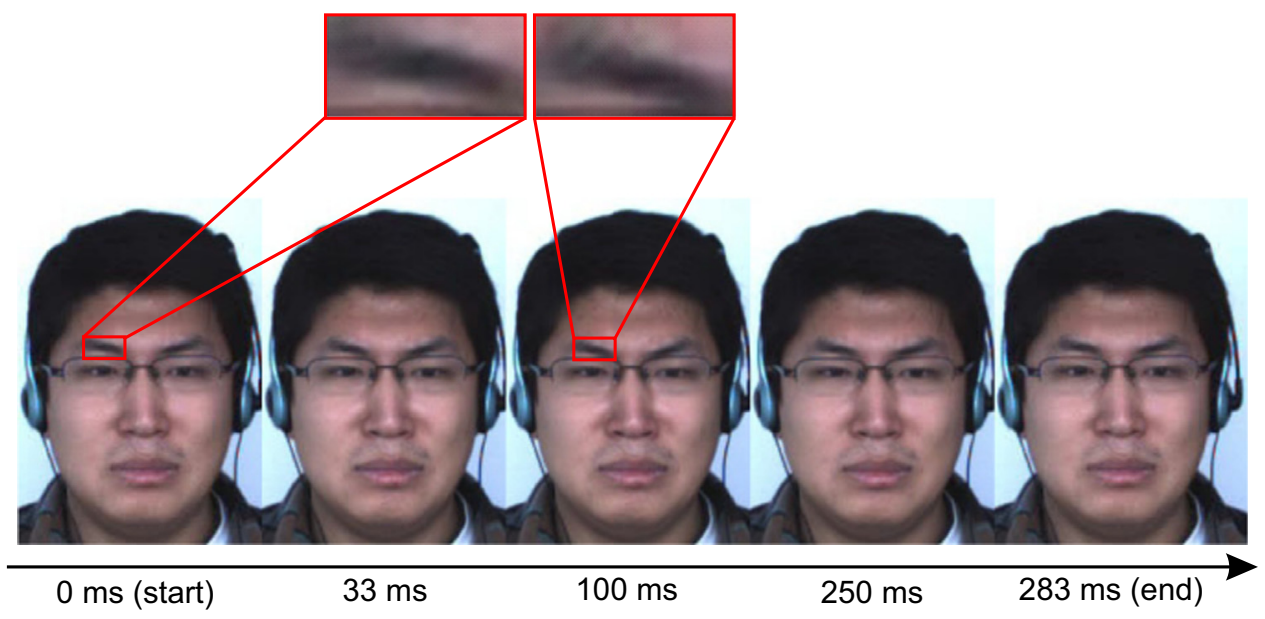

Fig. 3. A demonstration of the frame sequence of a micro-expression. The apex frame presents at about 100 ms. The AUs for this micro-expression are $4+10$, which indicates disgust. The movement is easier to detect in video play but not in a picture sequence.

Table 3

The following list shows the participants' ratings on the 17 video episodes, the main emotion for each episode, the number of participants who felt such an emotion and the corresponding mean score (from 0 to 6 ).

\begin{tabular}{llll}
\hline Episode & Main emotions & Rate of selection & Mean score \\
\hline 1 & Happiness & 0.69 & 3.27 \\
2 & Happiness & 0.71 & 3.6 \\
3 & Happiness & 0.7 & 3.14 \\
4 & Happiness & 0.64 & 4.43 \\
5 & Disgust & 0.81 & 4.15 \\
6 & Disgust & 0.69 & 4.18 \\
7 & Disgust & 0.78 & 4 \\
8 & Disgust & 0.81 & 3.23 \\
9 & Fear & 0.63 & 2.9 \\
10 & Fear & 0.67 & 2.83 \\
$11^{\mathrm{a}}$ & I & 1 & 1 \\
12 & Disgust (fear) & $0.60(0.33)$ & $3.78(0.28)$ \\
13 & Sadness & 0.71 & 4.08 \\
14 & Sadness & 1 & 5 \\
15 & Anger (sadness) & $0.69(0.61)$ & $4.33(0.62)$ \\
16 & Anger & 0.75 & 4.67 \\
17 & Anger & 0.94 & 4.93 \\
\hline
\end{tabular}

${ }^{\text {a }}$ No single emotion word was selected by one third of the participants.

captured in the database are relatively "pure and clear", without noises such as head movements and irrelevant facial movements.

(3) Action units were given for each micro-expression. AUs give detailed movements of facial expressions and help facilitate accurate emotion labeling $[16,17]$.

(4) The micro-expressions were carefully labeled based on psychological researches and participants' self-report. In addition, the unemotional facial movements were removed.

We recorded the facial expressions with two different environmental configurations and two different cameras. Therefore, we divide the samples into two classes: Class A and Class B. The samples in Class A were recorded by the BenQ M31 consumer camera with $60 \mathrm{fps}$, with the resolution set at $1280 \times 720$ pixels. The participants were recorded in natural light. The samples in Class B were recorded by the Point Grey GRAS-03K2C industrial camera with $60 \mathrm{fps}$, with the resolution set to $640 \times 480$ pixels. The participants were recorded in a room with two LED lights.

\subsection{Acquisition and coding}

In order to elicit "clear" micro-expressions, we employed the neutralization paradigm in which participants tried to keep their faces neutralized when experiencing emotions. We used video episodes as the eliciting material with contents that were considered high in emotional valence. In this study, the participants experienced high arousal and strong motivation to disguise their true emotions.

\subsubsection{Participants and elicitation stimuli}

35 Chinese participants ( 13 females, 22 males) were recruited with a mean age of 22.03 years (standard deviation $=1.60$ ) in the study. We used video episodes with high emotional valence as the elicitation materials. Seventeen video episodes were downloaded from the Internet, which were assumed to be highly positive or negative in valence and should elicit various emotions from the participants. The durations of the selected episodes ranged from about 1 min to roughly 4 min. Each episode mainly elicited one type of emotion. 20 participants rated the main emotions of the video episodes and scores from 0 to 6 were given to each, where 0 is the weakest and 6 the strongest (see Table 3 ).

\subsubsection{Acquisition procedure}

The neutralizing paradigm was used where participants tried to inhibit any facial movements with great efforts. Each participant was seated in front of a 19-inch monitor. The camera (Point Grey GRAS-03K2C or BenQ M31, with 60 frames per second) on a tripod was set behind the monitor to record the full-frontal face of the participants. The video episodes were presented on the screen which was controlled by the experimenter. The participants were told to closely watch the screen and maintain a neutral face. In addition, they were not allowed to turn their eyes or head away from the screen. After each episode was over, the participants were asked to watch their own facial movements in the recordings and indicated whether they produced irrelevant facial movements which could be excluded for later analysis.

\subsubsection{Data analysis}

To ensure the reliability, two coders were recruited to code the duration and AU-combination of the micro-expressions. They independently spot the onset, apex and offset frames, and arbitrated the disagreement. ${ }^{2}$ The reliability $R_{d}$ for duration calculation was 0.78 , which can be calculated by the following:

$R_{d}=\frac{2 \# f\left(C_{1} C_{2}\right)}{\# A l l \_ \text {frame }}$

${ }^{2}$ When they did not agree on the location, the average of the two coders numbers was taken. 
where $\# f\left(C_{1} C_{2}\right)$ is the number of frames on which Coder 1 and Coder 2 agreed and \#All_frame is the total number of frames scored by the two coders.

The reliability $R_{l}$ for AU labeling was 0.83 , which can be calculated by the following:

$R_{l}=\frac{2 \# A U\left(C_{1} C_{2}\right)}{\# \text { All } A U}$

where $\# A U\left(C_{1} C_{2}\right)$ is the number of AUs on which Coder 1 and Coder 2 agreed and \#All_AU is the total number of AUs scored by the two coders.

We analyzed the video recordings in the following steps:

Step 1: The first step was a rough selection. This procedure was to reduce the quantity of to-be-analyzed facial movements while not missing any possible micro-expressions. The coders played the recordings at half speed and roughly spot the onset, apex and offset frames and then selected the facial expressions that last less than $1 \mathrm{~s}$. It was also noticed that some of the leaked fast facial expressions in our study were characterized by a fast onset with a slow offset. Thus, fast-onset facial expressions with the onset phases $^{3}$ less than about $500 \mathrm{~ms}$ (though the total duration is longer than $1 \mathrm{~s}$ ) were selected for later analysis because of their special temporal features.

Step 2: The selected samples were then converted into pictures to facilitate spotting the subsequent steps.

Step 3: Habitual movements (such as blowing the nose) or other irrelevant movements (such as pressing the lips when swallowing saliva) were removed. These irrelevant facial movements were also confirmed by the participants after the recording session.

Step 4: By employing the frame-by-frame approach, the coders tried to spot the onset, apex and offset frames. Sometimes the facial expressions faded very slowly, and the changes between frames were very difficult to detect by eyes. For such offset frames, the coders only coded the last obvious changing frame as the offset frame while ignoring the nearly imperceptible changing frames.

\subsubsection{Micro-expression labeling}

Two coders labeled the micro-expression independently and then they arbitrated any disagreements. AUs were marked to give an objective and accurate description of the facial movements. Considering the differences in $\mathrm{AU}(\mathrm{s})$ combinations between microexpression and ordinary facial expression [8], emotion labeling cannot just rely on the certain $\mathrm{AU}(\mathrm{s})$ combination held for ordinary facial expressions. Therefore, we had to take into account participants' self-ratings and the content of the video episodes as well. Six basic emotions are unable to cover all the facial expressions. Thus, two additional classes of facial expressions were added: repression and tense. Repression occurs when people try to mask the true facial expressions by using certain muscles (such as AU 17) to repress, while tense indicates some kind of emotional responses without clear meaning. Though they are actually not emotions, they are useful in understanding people's genuine feelings. We set a little different criteria in labeling the microexpressions from the [11] as we further understood the emotional meaning of some $\mathrm{AU}(\mathrm{s})$ combinations (see Table 2).

\subsection{Database evaluation}

\subsubsection{Normalization}

The micro-expression samples were normalized both in spatial dimension and temporal dimension. For spatial dimension normalization, an upright frontal face image with regular features was

\footnotetext{
${ }^{3}$ The duration from the onset frame to the apex frame.
}

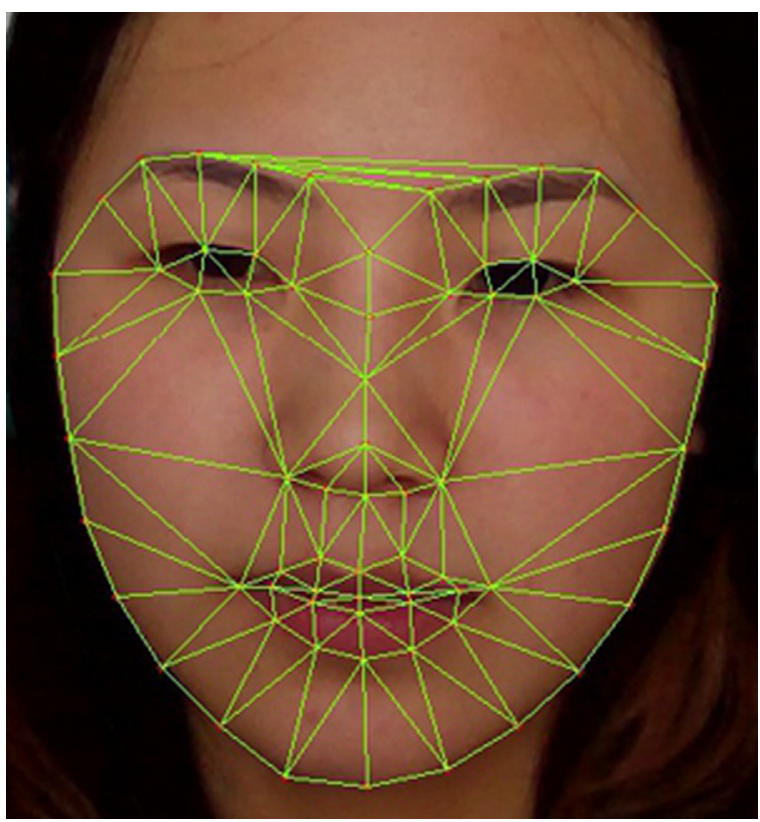

Fig. 4. An example face with its feature landmarks detected.

Table 4

Performance on recognizing 5-class micro-expressions with LBP-TOP feature extraction and leave-one-subject-out cross-validation method.

\begin{tabular}{lcc}
\hline Parameters & $5 \times 5$ & $8 \times 8$ \\
\hline$R_{x}=1, R_{y}=1, R_{t}=2$ & 59.67 & 59.12 \\
$R_{x}=1, R_{y}=1, R_{t}=3$ & 61.32 & 54.70 \\
$R_{x}=1, R_{y}=1, R_{t}=4$ & 57.46 & 53.04 \\
$R_{x}=2, R_{y}=2, R_{t}=2$ & 57.46 & 58.01 \\
$R_{x}=2, R_{y}=2, R_{t}=3$ & 58.56 & 54.70 \\
$R_{x}=2, R_{y}=2, R_{t}=4$ & 61.88 & 55.25 \\
$R_{x}=3, R_{y}=3, R_{t}=2$ & 55.25 & 60.22 \\
$R_{x}=3, R_{y}=3, R_{t}=3$ & 57.46 & 59.12 \\
$R_{x}=3, R_{y}=3, R_{t}=4$ & 59.12 & 59.12 \\
$R_{x}=4, R_{y}=4, R_{t}=2$ & 55.25 & 54.70 \\
$R_{x}=4, R_{y}=4, R_{t}=3$ & 55.80 & 55.25 \\
$R_{x}=4, R_{y}=4, R_{t}=4$ & 57.46 & 58.56 \\
\hline
\end{tabular}

selected as a template. The first frame of each micro-expression sample was marked with 68 landmarks by the Active Shape Model (ASM) [18] (see Fig. 4), which is a statistical model of the shape of objects which iteratively deform to fit to an example of the object in a new image. According to the 68 landmarks, the first frame of each sample was aligned to the template. There would be too much noise if all the frames were aligned to the template face by the 68 landmarks because the landmarks is not precisely and reliably labeled. We assumed that the micro-expressions were (mostly) not accompanied with head movements. Therefore, subsequent frames underwent the same transformation as the first frame. All the face images were cropped to the size at $163 \times 134$ pixels. For temporal dimension normalization, we used linear interpolation to normalize to 70 frames. Since microexpressions vary in duration (frames), we found the microexpression with the maximum frame number and made other micro-expressions normalized to that number.

\subsubsection{Method}

For feature extraction we used Local Binary Pattern histograms from Three Orthogonal Planes (LBP-TOP) [19] to describe the 
spatiotemporal local textures from the cropped face sequences. The radii in axes $X$ and $Y$ (be marked as $R_{x}$ and $R_{y}$ ) were assigned various values from 1 to 4 and the radii in axes $T$ (be marked as $R_{t}$ ) were assigned various values from 2 to 4 . The number of neighboring points (be marked as $P$ ) in the $X Y, X T$ and $Y T$ planes all was set as 4. SVM was used as the classifier. Since some types of samples are few, we only selected 5 classes of the facial expressions - happiness, surprise, disgust, repression and tense - for training and test. Considering the unequal distribution of types of samples and some types is only very few, leave-one-subject-out cross validation was employed. The LBP-TOP features were extracted in either $5 \times 5$ or $8 \times 8$ blocks. The performance of the two experiments is shown in Table 4 . The best performance is $61.88 \%$ when $R_{x}=2, R_{y}=2, R_{t}=3$ respectively in $5 \times 5$ blocks.

\section{Discussion and suggestions}

There exists several challenges in automatic micro-expression recognition. Here are some issues in using micro-expression database and developing effective recognition algorithms:

1. Normalization: Typically, all data need to undergo a normalization process. Many normalization methods have been developed for spatial dimension. However, very few studies explored the normalization process for temporal dimension. Since microexpressions vary in duration (frames), it is necessary to normalize the temporal dimension of micro-expressions. Though linear interpolation could be applied to this situation, many studies revealed that the facial images in the temporal dimension lie on a manifold. Several interpolation models based on manifolds have been developed, one such example is the Temporal Interpolation Model based on the Laplacian graph [14] which could be used to normalize the temporal dimension of micro-expression data.

2. Big data: Very high dimensional data are generated from a high-speed and high-resolution camera. A micro-expression video sequence of $0.5 \mathrm{~s}$, filmed at $200 \mathrm{fps}$, with a resolution of $800 \times 600$ would generate a data file of roughly $137 \mathrm{MB}$. Facing such big data, it is inappropriate to use methods such as PCA to project such high dimensional data into low dimensional spaces because it is hard to guarantee that all useful information would be preserved. Thus, it is necessary for researchers to develop more efficient algorithms.

3. AU coding: FACS [16] gives an objective method for quantifying facial movement in terms of component actions. Different groups may have different criteria for emotion classifications but could have the same AU coding system. Unlike posed facial expressions, for which people are asked to generate several preset facial movements, in our database micro-expressions were elicited by strong emotional stimuli. Therefore, it is thus inappropriate to forcefully classify these micro-expressions into six categories. For example, AU4 (frown) may indicate disgust, anger, attention or tense [8]. In this database we labeled AU4 as tense, a more general feeling. We believe this categorization is more plausible. ${ }^{4}$ Because of different emotion labeling criteria in different groups, we suggest that the to-be-developed automatic micro-expression recognition system should recognize $\mathrm{AUs}$ and then give an estimated emotion based on the extensive research of FACS.

4. Considering the temporal information: Some people may misunderstand micro-expression as ordinary facial expressions and tried to apply a current facial expression algorithm to microexpression. However, the partial and low-intensity facial movements in micro-expressions would differ from ordinary facial expressions and the computer may label the micro-expression as

\footnotetext{
${ }^{4}$ With these new understandings, the emotion labelings of the micro-
} expressions have now been updated in our database. a neutral face since the elicited facial expressions in this database were low in intensity. Without temporal information, microexpressions are much more difficult to detect. Thus, to better detect and recognize micro-expression, researchers should take temporal information into account.

5. Coding the duration: When developing a micro-expression database, manually spotting the onset, apex and offset frames is time- and effort-consuming. Because of this, very few research groups built spontaneous micro-expression databases. If some software is developed to help spot the onset, apex and offset frames, collecting micro-expression would be much easier. If such a software works, the reliability would be much higher compared to the manual work in which two coders judge the frames with somewhat different criteria, and such a difference may be large across different groups.

6. Some other concerns on micro-expression: Micro-expressions are not only fast but also subtle. Frame-by-frame scrutiny is usually more difficult than real-time observation to spot the micro-expressions. In another word, for human eyes, dynamic information is important in recognizing micro-expressions. In addition, the fast-onset facial expression should also be considered as facial expressions. Some of the facial expressions have fast onset but slow offset. These facial expressions, share the fundamental characteristics of micro-expressions, being involuntary, fast, and also revealing the genuine emotions that participants tried to conceal [8]. Therefore, we include these samples into the database as well.

\section{Conclusion and future work}

In conclusion, this paper briefly reviewed the previous microexpression databases and introduced how we built a microexpression database, CASME, with psychological guidance on an elicitation approach and emotional labeling. We provided a baseline evaluation for this database with LBP. Micro-expression recognition raised many challenges and we provided several suggestions that might help improve recognition rates of future algorithms. The full database file is available upon request to the corresponding author.

The database is small for the moment. Because elicitation of micro-expressions is not easy and manual coding is time-consuming, this database can only be enlarged bit by bit. We are trying to build a new micro-expression database, recruit more participants and elicit more micro-expressions. For the new version, which may be called CASME II, we will record the micro-expressions at $200 \mathrm{fps}$, with larger face size, and hopefully collect more microexpressions. With this micro-expression database, researchers may get higher accuracy due to the higher temporal and spatial resolution. And we can use CASME II to test whether higher resolution matters in recognition accuracy.

\section{Acknowledgments}

This work was supported in part by grants from 973 Program (2011CB302201), the National Natural Science Foundation of China (61075042, 61322206, and 61379095) and China Postdoctoral Science Foundation funded project (2012M580428). We appreciate Yu-Hsin Chen and Fangbing Qu's suggestions in language.

\section{References}

[1] P. Ekman, W. Friesen, Nonverbal leakage and clues to deception, Technical Report, DTIC Document, 1969.

[2] P. Ekman, Lie catching and microexpressions, in: The Philosophy of Deception, 2009, pp. 118-133. 
[3] M.G. Frank, M. Herbasz, K. Sinuk, A. Keller, A. Kurylo, C. Nolan, I See How You. Feel: Training Laypeople and Professionals to Recognize Fleeting Emotions. Paper presented at the annual meeting of the International Communication Association, Sheraton New York, New York City, NY, 2009.

[4] M. OSullivan, M. Frank, C. Hurley, J. Tiwana, Police lie detection accuracy: the effect of lie scenario, Law Hum. Behav. 33 (2009) 530-538.

[5] M. Frank, C. Maccario, V. Govindaraju, Behavior and Security, Greenwood Pub Group, Santa Barbara, California, 2009, pp. 86-106.

[6] E.A. Haggard, K.S. Isaacs, Methods of Research in Psychotherapy, AppletonCentury-Crofts, New York, 1966, pp. 154-165.

[7] P. Ekman, Darwin, deception, and facial expression, Ann. N. Y. Acad. Sci. 1000 (2006) 205-221.

[8] W.-J. Yan, Q. Wu, J. Liang, Y.-H. Chen, X. Fu, How fast are the leaked facial expressions: the duration of micro-expressions, J. Nonverbal Behav. (2013) $1-14$.

[9] D. Matsumoto, H. Hwang, Evidence for training the ability to read microexpressions of emotion, Motiv. Emot. 35 (2011) 181-191.

[10] G. Sandbach, S. Zafeiriou, M. Pantic, L. Yin, Static and dynamic 3d facial expression recognition: a comprehensive survey, Image Vis. Comput. (2012).

[11] W.J. Yan, Q. Wu, Y.J. Liu, S.J. Wang, X. Fu, Casme database: a dataset of spontaneous micro-expressions collected from neutralized faces, in: 10th IEEE Conference on Automatic Face and Gesture Recognition, 2013, Shanghai.

[12] M. Shreve, S. Godavarthy, D. Goldgof, S. Sarkar, Macro-and micro-expression spotting in long videos using spatio-temporal strain, in: IEEE Conference on Automatic Face and Gesture Recognition FG'11, IEEE, 2011, pp. 51-56.

[13] S. Polikovsky, Y. Kameda, Y. Ohta, Facial micro-expressions recognition using high speed camera and 3d-gradient descriptor, in: 3rd International Conference on Crime Detection and Prevention, ICDP 2009, IET, pp. 1-6.

[14] T. Pfister, X. Li, G. Zhao, M. Pietikainen, Recognising spontaneous facial microexpressions, in: 2011 IEEE International Conference on Computer Vision (ICCV), IEEE, 2011, pp. 1449-1456.

[15] G. Warren, E. Schertler, P. Bull, Detecting deception from emotional and unemotional cues, J. Nonverbal Behav. 33 (2009) 59-69.

[16] P. Ekman, W. Friesen, J. Hager, FACS Investigators Guide, A Human Face, 2002.

[17] M. Bartlett, G. Littlewort, M. Frank, C. Lainscsek, I. Fasel, J. Movellan, Automatic recognition of facial actions in spontaneous expressions, J. Multimed. 1 (2006) 22-35.

[18] T.F. Cootes, C.J. Taylor, D.H. Cooper, J. Graham, Active shape models-their training and application, Comput. Vis. Image Underst. 61 (1995) 38-59.

[19] G. Zhao, M. Pietikainen, Dynamic texture recognition using local binary patterns with an application to facial expressions, IEEE Trans. Pattern Anal. Mach. Intell. 29 (2007) 915-928.

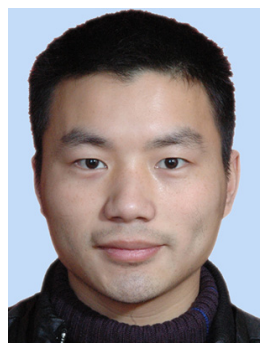

Wen-Jing Yan was born in 1985 in Wenzhou, China. He is a doctoral student in Institute of Psychology, Chinese Academy of Sciences. His interests include facial expression (especially micro-expression) and deception. He is now trying interdisciplinary researches among behavioral science, affective computing and pattern recognition.

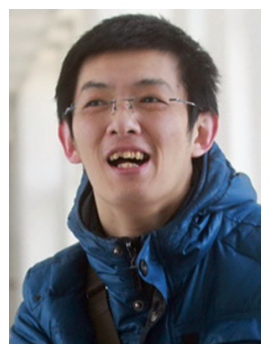

Su-Jing Wang received the Master's degree from the Software College of Jilin University, Changchun, China, in 2007. He received the Ph.D. degree from the College of Computer Science and Technology of Jilin University in 2012. He is a postdoctoral researcher in Institute of Psychology, Chinese Academy of Sciences. He has published more than 30 scientific papers. He is One of the Ten Selectees of the Doctoral Consortium at International Joint Conference on Biometrics 2011. He was called as Chinese Hawkin by the Xinhua News Agency. His research was published in IEEE Transactions on Image Processing, IEEE Transactions on Neural Networks and Learning Systems, Neurocomputing, etc. His current research interests include pattern recognition, computer vision and machine learning. He also reviews for several top journals, such as IEEE Transactions on Pattern Analysis and Machine Intelligence. Currently, he is an Associate Editor of Neurocomputing.

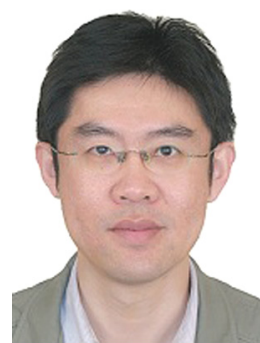

Yongjin.htm.
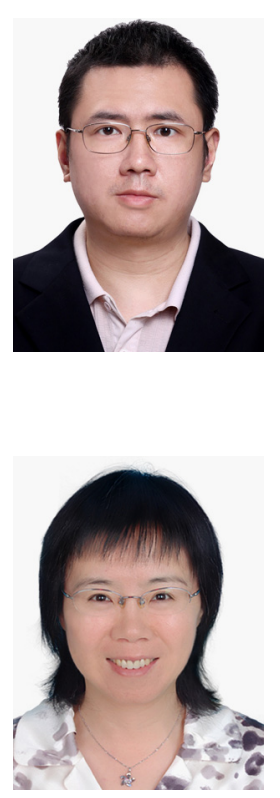

Yong-Jin Liu received his B.Eng. degree from Tianjin University, China, in 1998, and his Ph.D degree from the Hong Kong University of Science and Technology, Hong Kong, China, in 2003. He is now an Associate Professor with the Tsinghua National Laboratory for Information Science and Technology, Department of Computer Science and Technology, Tsinghua University, China. His research interests include pattern recognition, computer graphics, computational geometry and computer-aided design. He is a member of IEEE, a member of IEEE Computer Society and IEEE Communications Society. More research topics about him can be found at http://cg.cs.tsinghua.edu.cn/people/ Yongjin/

Qi Wu received his Ph.D degree from Institute of Psychology, Chinese Academy of Sciences, Beijing, China, in 2012. He is now an Assistant Processor with Department of Psychology, Hunan Normal University, China. His research interests include affective computing and social cognition.

Xiaolan Fu was born in 1963. She received her Ph.D. degree in 1990 from Institute of Psychology, Chinese Academy of Sciences. Currently, she is a Senior Researcher at Cognitive Psychology. Her research interests include Visual and computational cognition: (1) attention and perception, (2) learning and memory, and (3) affective computing. At present, she is the director of Institute of Psychology, Chinese Academy of Sciences and Vice Director, State Key Laboratory of Brain and Cognitive Science. 